\title{
DIAGNÓSTICO DOS ANEURISMAS CEREBRAIS POR ANGIOTOMOGRAFIA TRIDIMENSIONAL
}

\author{
Guilherme Cabral de Andrade1, Pedro Augusto Deja Teixeira', \\ Helvércio Fernando Polsaque Alves'2, Erik Evandro Donato ${ }^{3}$, \\ Carlos Alexandre Aguiar Moreira ${ }^{4}$, Renato Campos Soares de Faria ${ }^{5}$, \\ Luís Antônio Araújo Dias ${ }^{6}$
}

\begin{abstract}
RESUMO - A angiotomografia é método diagnóstico rápido e minimamente invasivo que passou a ser utilizado recentemente nos casos de aneurisma intracraniano, possuindo $100 \%$ de especificidade e podendo atingir $95 \%$ de sensibilidade, na avaliação dos aneurismas do círculo de Willis, substituindo a angiografia digital, mas não em todos os casos. Apresentamos nossa experiência na avaliação do exame de angiotomografia tridimensional em período de 2 anos, comprendido entre junho de 1997 e junho 1999, em que foram realizados exames de angiotomografia tridimensional para detecção de aneurismas intracranianos em 136 pacientes Em 118 pacientes foi realizada apenas angiotomografia e em 18 casos foi realizada além da angiotomografia tridimensional, também a angiografia digital convencional. 0 exame de angiotomografia tridimensional possui baixo risco, sendo melhor utilizada na detecção dos aneurismas com diâmetro maior que $3 \mathrm{~mm}$. Possui algumas limitações, como os aneurismas muito pequenos $<3 \mathrm{~mm}$ aneurismas da carótida cavernosa e da circulação posterior, como também a não realização do exame dinâmico.
\end{abstract}

PALAVRAS-CHAVE: aneurisma cerebral, angiotomografia tridimensional.

\begin{abstract}
Cerebral aneurysms diagnosis by three-dimensional CT angiography
ABSTRACT - Three-dimensional computerized tomography angiography is a fast diagnosis method and low invasive that passed to be used recently in the cases of intracranial aneurysms. This method presents a $100 \%$ specificity and can reach $95 \%$ of sensibility in the evaluation of the aneurysms in the circle of Willis, substituting the digital angiography but not in all of the cases. We report our experience in evaluation of the threedimensional angiotomography exams in a period of 2 years between June of 1997 and June of 1999, were accomplished for detection of intracranial aneurysms in 136 patients, being 118 just with three-dimensional angiotomography and in 18 cases they were accomplished besides three-dimensional angiotomography also the conventional digital angiography. The exam of three-dimensional angiotomography possesses low risk being used better in the detection of the aneurysms when possess higher diameter than $3 \mathrm{~mm}$, with some limitations as in the very small aneurysms when smaller than $3 \mathrm{~mm}$, carotid intern cavarnous aneurysms, posterior circulation aneurysms as well as the non accomplishment of the dynamic exam.
\end{abstract}

KEY WORDS: cerebral aneurysm, computerized three-dimensional, angiotomography.

Aneurismas cerebrais e hemorragia subaracnóidea possuem elevado índice de morbi-mortalidade. O seu diagnóstico precoce é um dos fatores que possibilita a boa evolução da doença. $O$ diagnóstico dos aneurismas intracranianos passou a ser realizado com o advento da angiografia cerebral apresentada à comunidade científica por Egas Moniz em julho de $1927^{1}$. Ainda hoje, o exame "padrão ouro" para a detecção dos aneurismas intracranianos é a angiografia. Nos últimos anos surgiram duas novas modalidades diagnósticas para as patologias vasculares intracranianas: a angiotomografia tridimensional e a angio-ressonância magnética. $\mathrm{O}$ uso da tomografia com infusão de contraste, nos casos em que há suspeita de aneurisma intracraniano, pode detectar, dependendo da localização, de $36 \%$ no complexo

\footnotetext{
Serviço de Neurocirurgia/Neurorradiologia da Santa Casa de Ribeirão Preto SP, Brasil: ${ }^{1}$ Pós Graduando da Universidade Federal de São Paulo - Escola Paulista de Medicina (UNIFESP/EPM-SP); ${ }^{2}$ Especializando em Dor e Neurocirurgia Funcional Hospital AC Camargo SP; ${ }^{3}$ Residente; ${ }^{4}$ Preceptor; ${ }^{5}$ Chefe do Serviço de Neurorradiologia; ${ }^{6}$ Chefe do Serviço de Neurocirurgia.
}

Recebido 19 Junho 2002, recebido na forma final 23 Agosto 2002. Aceito 12 Setembro 2002.

Dr. Guilherme Cabral de Andrade - Avenida Dr. Altino Arantes 865/62 - 04042-000 São Paulo SP - Brasil.E-mail:cabralnec@uol.com.br 
carótida interna até $76 \%$ na artéria comunicante anterior e artéria cerebral média ${ }^{2}$, com média de $57 \%$ no polígono de Willis ${ }^{3}$, havendo maior sensibilidade para os aneurismas com tamanho $>7 \mathrm{~mm}$, principalmente quando há presença de calcificação ou massa com aumento de densidade nas cisternas basais. Porém, ramos arteriais ou "loops" podem simular pequenos aneurismas ${ }^{4,5} \mathrm{com}$ a necessidade da complementação do estudo. Vasos de até $1,5 \mathrm{~mm}$ de diâmetro também podem ser vistos neste tipo de estudo ${ }^{6}$. As principais causas de dificuldade de visualização de aneurismas no estudo tomográfico com infusão de contraste são: 1) posicionamento inadequado do paciente; 2) baixa velocidade de infusão do contraste; 3) vasoespasmo ; 4) artefatos ${ }^{3}$. $\mathrm{O}$ primeiro relato na literatura com o uso de tomografia espiral foi realizado por Kalender ${ }^{7}$. Os primeiros estudos experimentais com angiotomografia helicoidal foram realizados com reconstrução de artéria aorta ${ }^{8}$, artéria renal, esplênica e de membros inferiores $^{9,10}$ e outras áreas, incluindo as artérias extracraniais $^{11-13}$.

O uso da angiotomografia helicoidal ou espiral com reconstrução em três dimensões é técnica de diagnóstico dos aneurismas intracranianos recentemente adotada, sendo grande aliado na estratégia terapêutica destes. Vem firmando-se como opção no diagnóstico dos aneurismas intracranianos. Apresentamos nossa experiência.

\section{MÉTODO}

De junho de 1997 a junho de 1999, foram estudados 262 pacientes com hemorragia subaracnóidea, a qual foi causada por aneurisma em 244 pacientes. Do total, 118 (41\%) pacientes foram investigados apenas com angiotomografia, 18 pacientes $(7,4 \%)$ foram investigados com angiotomografia e angiografia digital convencional, e, no restante, 126 $(51,6 \%)$ usou-se a angiografia digital apenas.

Os exames de angiotomografia foram realizados com o aparelho de tomografia helicoidal da Picker International (PG 5000), com cortes tomográficos axiais de $3 \mathrm{~mm}$. Após a punção venosa periférica com Abocath $20^{R}$ e conexão do mesmo a uma bomba mecânica de infusão de contraste Liebel-Flarsheim-Company (ANGIOMAT 6000), são injetados cerca de $120 \mathrm{cc}$ de contraste iodado hidrossolúvel (lopamiron ${ }^{\mathrm{R}}$ ), levando-se um tempo médio de 8 minutos até a realização completa do exame. Em todos os pacientes que foram submetidos ao diagnóstico com angiotomografia, o exame foi realizado na admissão, imediatamente após ter sido confirmado o diagnóstico de hemorragia subaracnóidea nos cortes convencionais de tomografia, submetendo-o de imediato ao protocolo de angiotomografia. Após realização do exame, as imagens foram transferidas para uma estação de trabalho Picker ${ }^{R}$, para reconstrução do sistema arterial em três dimensões (Fig 1),
Tabela 1. Resultados concluidos após estudo da casuística, em que foram avaliados 136 pacientes com aneurisma intracraniano por angiotomografia $3 D$.

\begin{tabular}{ll}
\hline Colo aneurismático & boa identificação \\
Rotação em 3D & possível \\
Angioscopia virtual & possível \\
Complicações & infrequentes \\
Teste de Compressão & impossível \\
Visualização de & regular \\
vasos menores & \\
Circulação posterior & regular \\
Acurácia diagnóstica & boa (aneurismas >3mm) \\
Vasoespasmo & regular \\
Relações atômicas & eficaz \\
Localização & bom \\
Sistema venoso & possível \\
Custo & $1 / 3$ da convencional \\
\hline
\end{tabular}

correlacionando-o com estruturas ósseas, permitindo rotações em $360^{\circ}$ (Fig 2 e 3), como também mensuração do diâmetro do colo do aneurisma (Fig 4). Todos os exames eram discutidos com neurocirurgiões juntamente com um neurorradiologista com experiência no método diagnóstico e, em algumas situações onde havia dúvida da localização e relações anatômicas do aneurisma, houve acompanhamento intra-operatório do neurorradiologista com o intuito de melhorar a precisão do método.

\section{RESULTADOS}

Os resultados são apresentados na Tabela 1.

\section{DISCUSSÃO}

O diagnóstico dos aneurismas intracranianos ainda possui como método "padrão ouro" a angiografia digital através da técnica de Seldinger, com cateterização da artéria femoral e estudo dos quatro grandes vasos intracranianos, possuindo elevada especificidade e sensibilidade. Tem a grande vantagem de ser realizado o estudo dinâmico da vascularização intracraniana, porém, como todo método invasivo, possui riscos de complicações que variam entre $0,1 \%$ e $2,6 \%$ em pacientes saudáveis, com déficit neurológico permanente em $0,33 \%$ dos pacientes submetidos ao estudo angiográfico nas mais diversas patologias e de $0,63 \%$ nos pacientes com doenças cérebro-vasculares ${ }^{14}$. Em estudo comparativo entre angiografia convencional e angioto- 


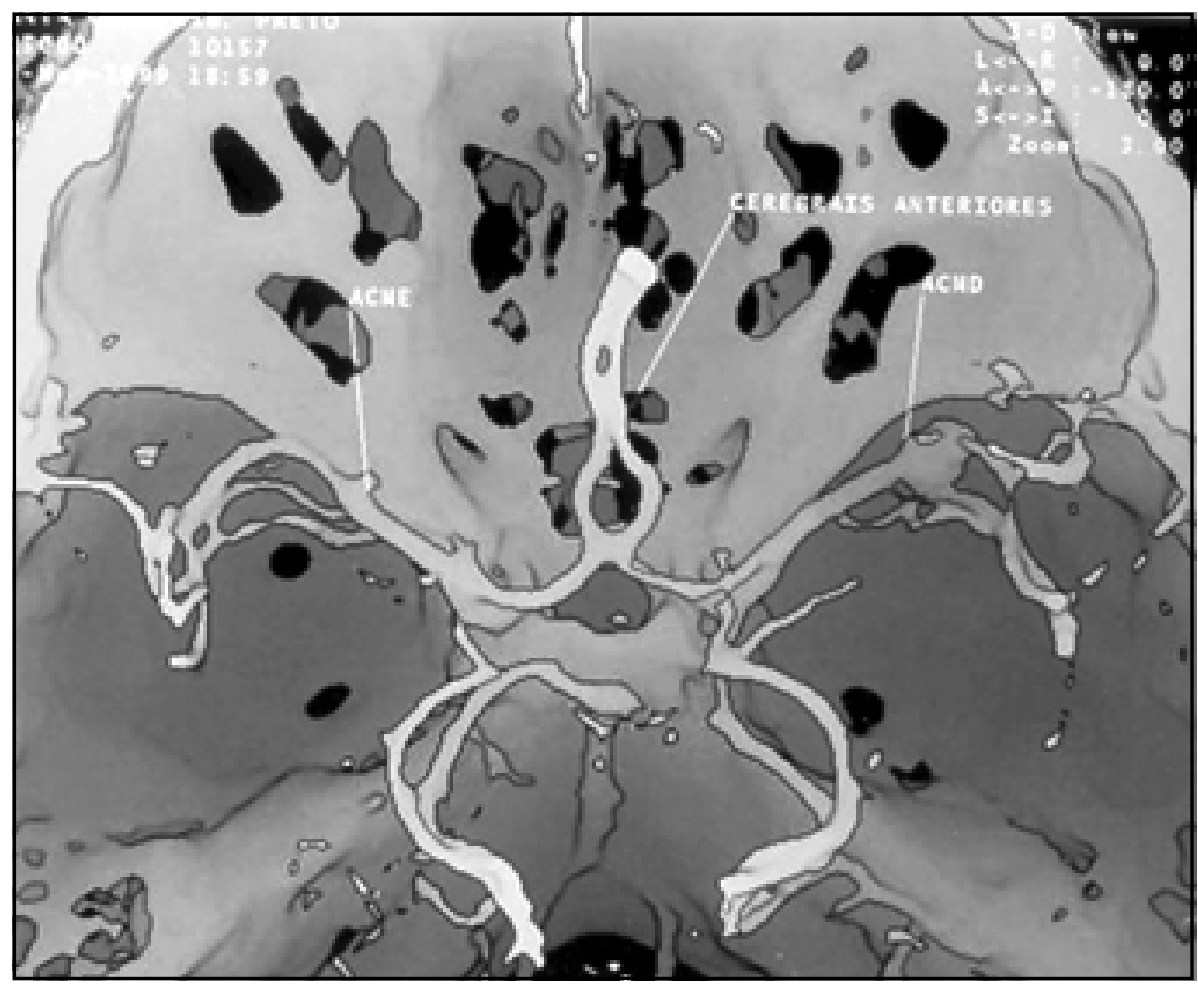

Fig 1. Reconstrução tridimensional das artérias do polígno de Willis, juntamente com estruturas ósseas da base do crânio.

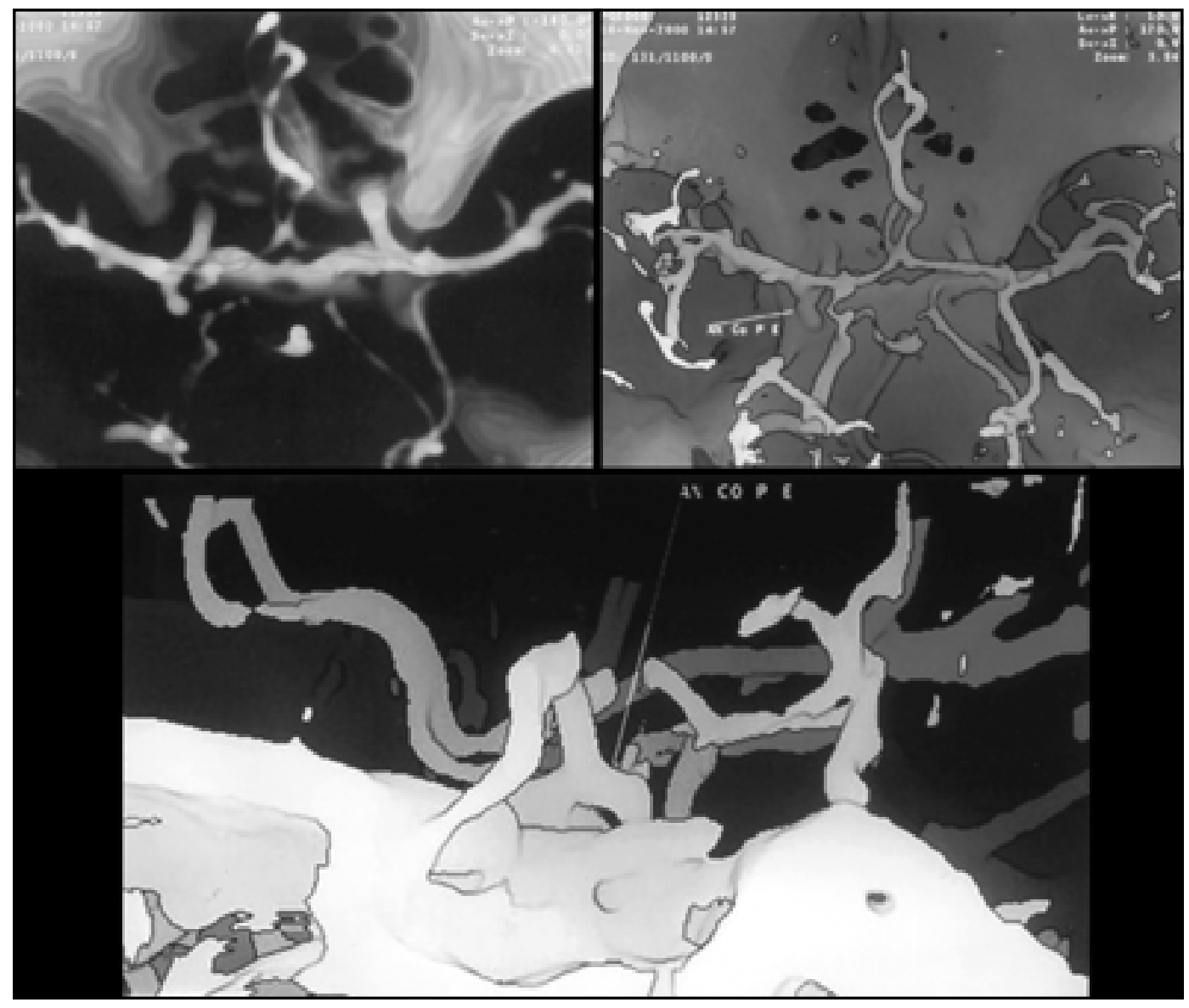

Figs 2 e 3. Reconstrução tridimensional de um aneurisma da artéria comunicante posterior esquerda, correlacionamento com estruturas ósseas, com visão superior e lateral do aneurisma. 


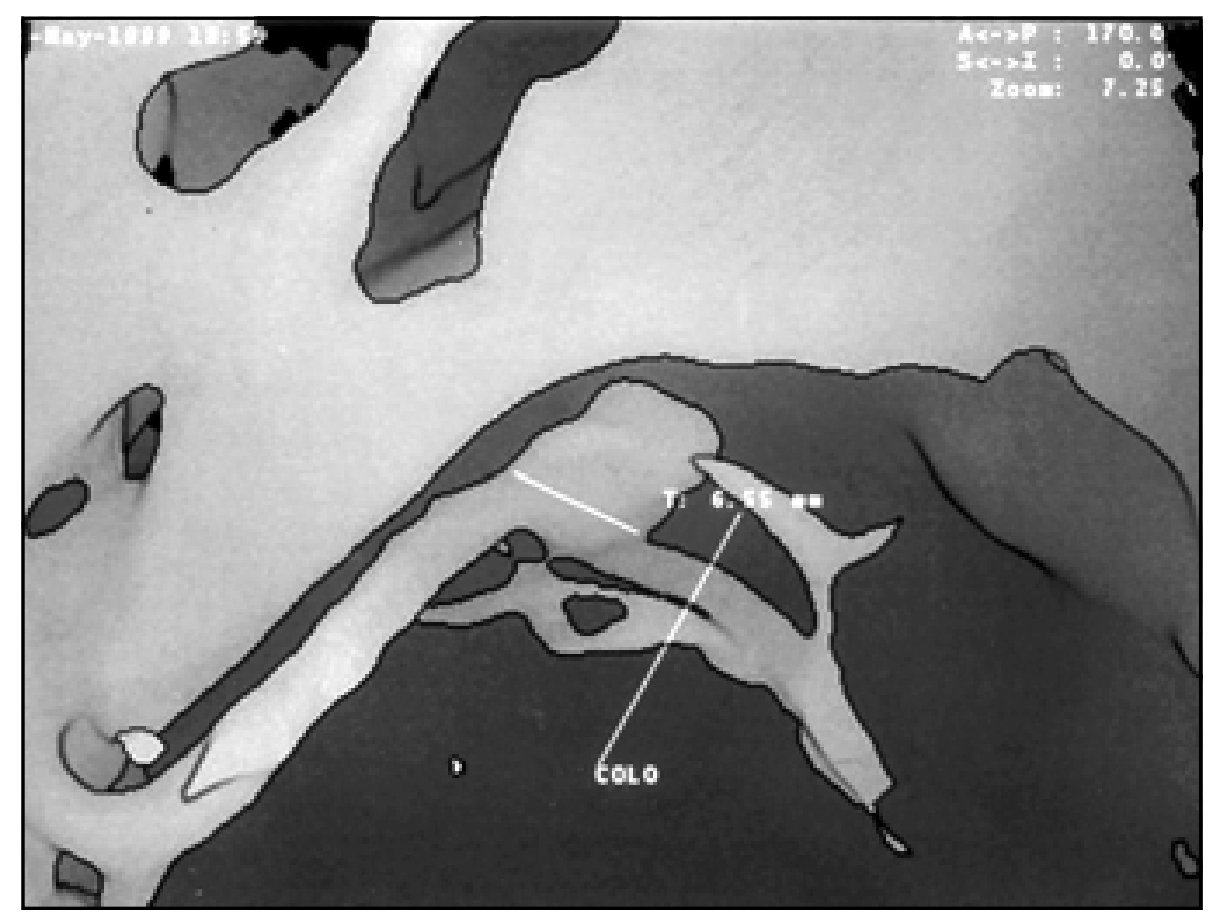

Fig 4. Exemplo da mensuração de um colo aneurismático em aneurisma da artéria cerebral média.

mografia $^{15}$, é vista como vantagem do método a possibilidade de reconstrução tridimensional da arquitetura dos vasos do polígono de Willis, permitindo rotação de até $360^{\circ}$, além de relacionar o aneurisma com as estruturas ósseas adjacentes, o que possibilita ao neurocirurgião melhor precisão no entendimento das relações do aneurisma e estruturas ósseas em torno deste, o que poderá influenciar na escolha da abordagem. Outra vantagem do método é o diagnóstico de calcificações no colo aneurismático, quando presentes ${ }^{16-18}$. Outra vantagem do método é a rapidez, com a necessidade de punção venosa periférica apenas, diminuindo os riscos de complicações, inerentes ao uso de contraste iodado. Segundo Yamamoto e cols ${ }^{19}$, a dose mínima de contraste iodado utilizada para a realização do exame é de $1 \mathrm{ml} / \mathrm{kg}$ com velocidade de infusão de $2 \mathrm{ml} / \mathrm{s}$, o que possibilita uma concentração de contraste sanguíneo na artéria carótida de mais de $15 \mathrm{mg} / \mathrm{ml}$ por tempo estimado de 20 segundos. Isto permite obter imagens do polígono de Willis, veias profundas e corticais.

Dusch e cols ${ }^{20}$ dividiram os pacientes em grupos clínicos definidos para a realização de angiotomografia: grupo 1) avaliação de pacientes mostraram lesões suspeitas de aneurisma intracraniano em exame de tomografia convencional, após investigação de cefaléia ou outros sintomas; grupo 2) seguimento dos pacientes com aneurismas intracranianos não tratados, avaliando aumento de seu tamanho; gru- po 3) seguimento dos aneurismas tratados mas que apresentaram colo residual após o tratamento; grupo 4) diagnóstico de aneurismas em pacientes com hemorragia subaracnóidea e angiografia convencional normal; grupo 5) screening para famílias com história de aneurisma intracraniano e para pacientes com passado de aneurisma.

A angiotomografia tridimensional possui sensibilidade em torno de $95 \%$ para todos os aneurismas intracranianos com tamanho acima de $2 \mathrm{~mm}^{21-25}$. Para os aneurismas da artéria comunicante anterior, a angiotomografia tridimensional mostra-se superior à angiografia convencional ${ }^{24,26}$.

As limitações do método são: 1) a não visualização de vasos com o diâmetro menor que $1 \mathrm{~mm}$, como as artérias perfurantes, artéria coroidéia anterior, artéria recorrente de Heubner; 2 ) dificuldade na discriminação entre artéria e veia, quando demonstradas simultaneamente; 3) não informação da dinâmica vascular; 4) aneurismas localizados na base do crânio, como os aneurismas da carótida cavernosa ${ }^{23}$.

A avaliação pós operatória dos pacientes submetidos ao tratamento cirúrgico dos aneurismas intracranianos, com a clipagem do aneurisma, pode ser realizada com o uso da angiotomografia em 3D, avaliando: a clipagem total ou não do colo aneurismático; a oclusão inadivertida de vasos na periferia do aneurisma e colo aneurismático; a permanência de fluxo sanguíneo no interior do aneurisma ${ }^{27}$. 
$\mathrm{Na}$ avaliação do exame de angiotomografia em 3D é de fundamental importância a participação do neurocirurgião juntamente com o neurorradiologista com experiência no método ${ }^{18}$.

\section{CONCLUSÃO}

A angiografia digital convencional ainda é o método "padrão ouro" na avaliação dos aneurismas intracranianos. Hoje, o arsenal diagnóstico possui outros métodos investigativos, porém todos eles colocam-se como estudos complementares, não podendo substituir a angiografia digital convencional. A angiotomografia tridimensional é um novo método diagnóstico que vem sendo usado nos últimos anos, trazendo novas informações na investigação dos aneurismas intracranianos. Tem como principais vantagens a rapidez, pouca invasividade, menor risco de complicações, menor custo; fornece ainda detalhes que não são vistos na angiografia digital, podendo diminuir os riscos de morbi-mortalidade inerentes à patologia. Em virtude das vantagens encontradas no método, todos os casos de hemorragia subaracnóidea admitidos no serviço são submetidos a investigação inicial com angiotomografia em 3D; em grande quantidade de casos fornece subsídios para definição do tratamento, mesmo sem a ajuda da angiografia digital. Outro fator importante é a experiência do neurorradiologista com o método, por não ser ainda utilizado de maneira generalizada.

\section{REFERÊNCIAS}

1. Mayberg MR, Winn R. The history of neurosurgical technique. Neurosurgery Clinics of North America. Philadelphia: Saunders,2001:1-228.

2. Ghoshhajra K, Scotti L, Marasco J, Baghai-Naiini P.CT detection of intracranial aneurysms in subarachnoid hemorrhage. AJR 1979;132:613-616.

3. Katada K, Kanno T, Sano H, Shibata T, Toda T, Koga S. CT in evaluation of the circle of Willis. Neuroradiology 1978;16:337-339.

4. Yamamoto Y, Asari S, Sunami N, Kunishio K, Fukui K, Sadamoto K. Computed angiotomography of unruptured cerebral aneurysms. J Comput Assist Tomogr 1986;10:21-27.

5. Asari S, Satoh T, Sakurai M, Yamamoto Y, Sadamoto K. Delineation of unruptured cerebral aneurysms by computerized angiotomography. J Neurosurg. 1982;57:527-534.

6. Weinstein MA, Duchesneau PM, Weinstein AC. Computed angiotomography. Am J Roentgenol.1977;129:699-701.
7. Kalender WA,Seissler W,Klotz E,Vock P.Spiral volumetric CT with single-breath-hold techinique, continuous transport, and continuous scanner rotation. Radiology 1990;176:181-183.

8. Costello P, Ecker CP, Tello R, Hartnell GG. Assessment of the thoracic aorta by spiral CT. AJR 1992;158:127-130.

9. Dillon EH, van Leeuwen MS, Fernandez MA, Mali WPTM. Spiral CT angiography. AJR 1993;160:1273-1278.

10. Galanski M, Prokop M, Chavan A, Schaefer CM, Jandeleit K, Nischelsky JE. Renal arterial stenoses: spiral CT angiography.Radiology 1993;189:185-192.

11. Dillon EH, van Leeuwen MS,Fernandez MA,Eikelboom BC, Mali WPTM. CT angiography: application to the evaluation of carotid artery stenosis. Radiology 1993;189:211-219.

12. Marks MP, NapelS, Jordan JE, Enzmann DR. Diagnosis of carotid artery disease: preliminary experience with maximum-intensity-projection spiral CT angiography. AJR 1993;160:1267-1271.

13. Schwartz RB, Jones KM, Chernoff DM, et al. Common carotid artery bifurcation: evaluation with spiral CT. Radiology 1992;185:513-519.

14. Earnest F IV, Forbes G, Sandok BA, et al. Complications on cerebral angiography prospective assessment of risk. AJR 1984;14:247-253.

15. Schwartz RB, Tice HM, Hooten SM, Hsu RTL, Stieg PE. Evaluation of cerebral aneurysms with helical CT: correlation with conventional angiography and MR angiography. Radiology 1994;192:717-722.

16. Zouaoui A, Sahel M, Marro B, et al. Three-dimensional computed tomography angiography in detection of cerebral aneurysms in acute subarachnoid hemorrhage. Neurosurg. 1997;41:125-130.

17. Ogawa T, Okudera T, Noguchi K, et al. Cerebral aneurysms: evaluation with three-dimensional CT angiography. AJNR 1996;17:447-454.

18. Anderson GB,Steinke DE,Petruk KC et al.Computed tomography angiography versus digital subtraction angiography for the diagnosis and early treatment of ruptured intracranial aneurysms. Neurosurg 1999;45:1315-1322.

19. Yamamoto $Y$, Satoh T, Masaru S, Asari S, Sadamoto K. Minimum dose contrast bolus in computed angiotomography of the brain. J Comput Assist Tomogr 1982;6:575-585.

20. Dorsch NWC, Young N, Kingston RJ, et al. Early experience with spiral CT in the diagnosis of intracranial aneurysms. Neurosurg 1995;36:230-238.

21. Alberico RA, Patel M, Casey S, et al. Evaluation of the circle of Willis with three-dimensional CT angiography in patients with suspected intracranial aneurysms. AJNR 1995;16:1571-1580.

22. Velthuis BK, van Leeuwen MS, Witkamp TD, et al. Computerized tomography angiography in patients with subarachnoid hemorrhage: from aneurysm detection to treatment without conventional angiography. J Neurosurg 1999;91:761-767.

23. Matsumoto M, Satoh N, Kobayashi T, et al. Helical CT for emergency patients with cerebrovascular disease-diagnosis of cerebral aneurysms with subarachnoid hemorrhage (SAH) by three-dimensional CT angiography (3-DCTA). Surg Cerebral Stroke 1996;24:177-185.

24. Strayle-Batra M, Skalej M, Wakhloo AK, et al. Three-dimensional spiral CT angiography in the detection of cerebral aneurysms. Acta Radiol 1998;39:233-238.

25. Hope JKA, Wilson JL, Thomson FJ. Three-dimensional CT angiography in the detection and characterization of intracranial Berry aneurysms. AJNR 1996;17:439-445.

26. Hashimoto H, Iida J, Hironaka $Y$, et al. Use of spiral computerized tomography angiography in patients with subarachnoid hemorrhage in whom subtration angiography did not revealed cerebral aneurysms. J Neurosurg 2000;92:278-283.

27. Vieco PT, Morin EE III, Gross CE. CT angiography in the examination of patients with aneurysm clips. AJNR 1996;17:455-457. 